\title{
A Novel Atypical Presentation of Frontal Fibrosing Alopecia Involving the Frontoparietal Scalp
}

\author{
Chloe Goldman ${ }^{a} \quad$ Aisleen Diaz ${ }^{b}$ Mariya Miteva ${ }^{a}$
}

aDr. Phillip Frost Department of Dermatology and Cutaneous Surgery, University of Miami L. Miller School of Medicine, Miami, FL, USA; 'bepartment of Dermatology, Ponce Health Sciences University School of Medicine, Ponce, PR, USA

\section{Established Facts}

- Frontal fibrosing alopecia is an irreversible scarring alopecia that classically presents with frontotemporal hairline recession and eyebrow loss.

- Over the past 2 decades, there has been an increasing incidence of frontal fibrosing alopecia as well as increased recognition of atypical clinical presentations.

\section{Novel Insights}

- We describe a case of frontal fibrosing alopecia presenting in an "upsilon"-shaped symmetric pattern of hair loss.

- Clinicians should be aware of this pattern as it closely mimics traction alopecia.

\section{Keywords}

Frontal fibrosing alopecia $\cdot$ Hair loss $\cdot$ Scarring $\cdot$ Scalp

\begin{abstract}
Frontal fibrosing alopecia (FFA) is a progressive scarring alopecia of unknown etiology that classically presents with a band of hair loss along the frontotemporal scalp. We report a case of FFA involving a band of alopecia along the frontotemporal scalp extending into 2 symmetrical triangles along the parietal scalp reminiscent of the Greek letter upsilon (u). Trichoscopy demonstrated loss of follicular ostia and peripilar casts. Histology demonstrated altered follicular architecture with decreased follicular density and focal perifollicular fibrosis with a lichenoid infiltrate. Both the trichoscopy and histology support a diagnosis of FFA. @ 2020 S. Karger AG, Basel
\end{abstract}

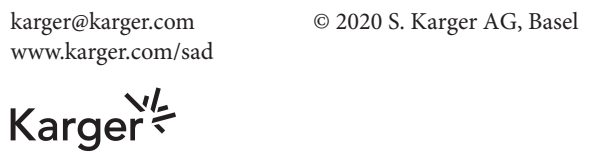

\section{Introduction}

Frontal fibrosing alopecia (FFA) is an irreversible scarring alopecia that typically affects postmenopausal women $[1,2]$. Its etiology remains unknown; however, previous studies have proposed a hormonal imbalance and genetic factors may play a role [3-5]. Most common clinical characteristics include a band-like pattern of scarring alopecia involving the frontal and temporal scalp, body hair loss, and, in a majority of cases, preceding eyebrow hair loss $[3,6,7]$.

A recent classification of FFA described 3 typical clinical patterns: (1) a linear uniform band of hair loss of the frontal hairline (most common), (2) a diffuse or zigzag pattern at and behind the frontal hair line, and (3) a "pseudo-fringesign," presenting with hair loss behind the preserved frontal 

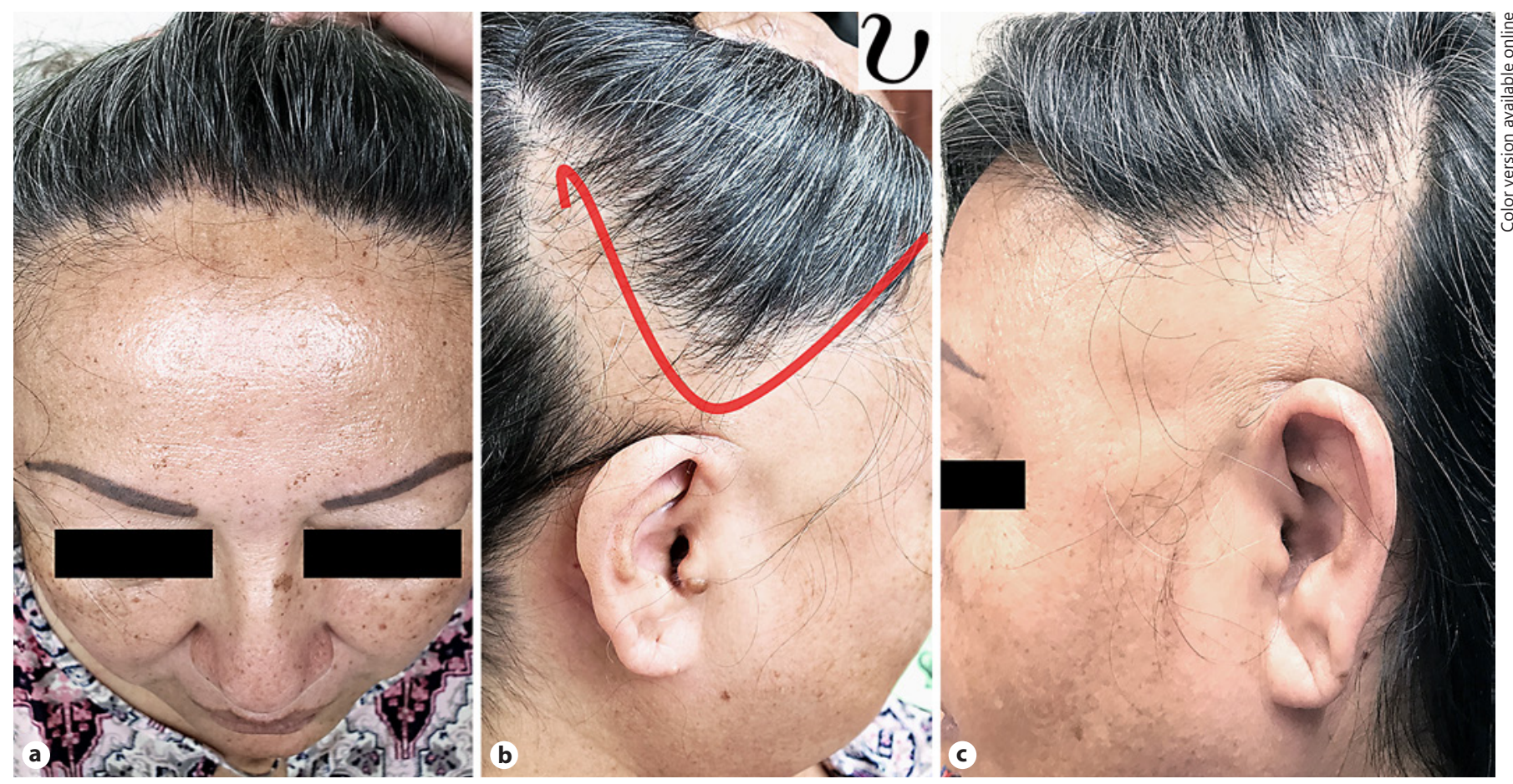

Fig. 1. Frontal fibrosing alopecia. Frontal and temporal hairline recession with bilateral eyebrow hair loss and lonely hairs (a); the alopecic areas are extending into 2 symmetrical triangles along the parietal scalp, resembling an upsilon (v) pattern (depicted by red upsilon shaped outline); right view (b) and left view (c).

Fig. 2. Trichoscopy. Findings show loss of follicular ostia, peripilar hypopigmented halos, and transparent emergences of the proximal hair shafts (black arrows) and pili torti like hairs (red arrow) on the temple (a) (FotoFinder Dermoscope, $\times 20$ ). Focal peripilar casts (black arrows) and absence of peripilar erythema on the frontal hairline (b) (FotoFinder Dermoscope, $\times 20$ ).
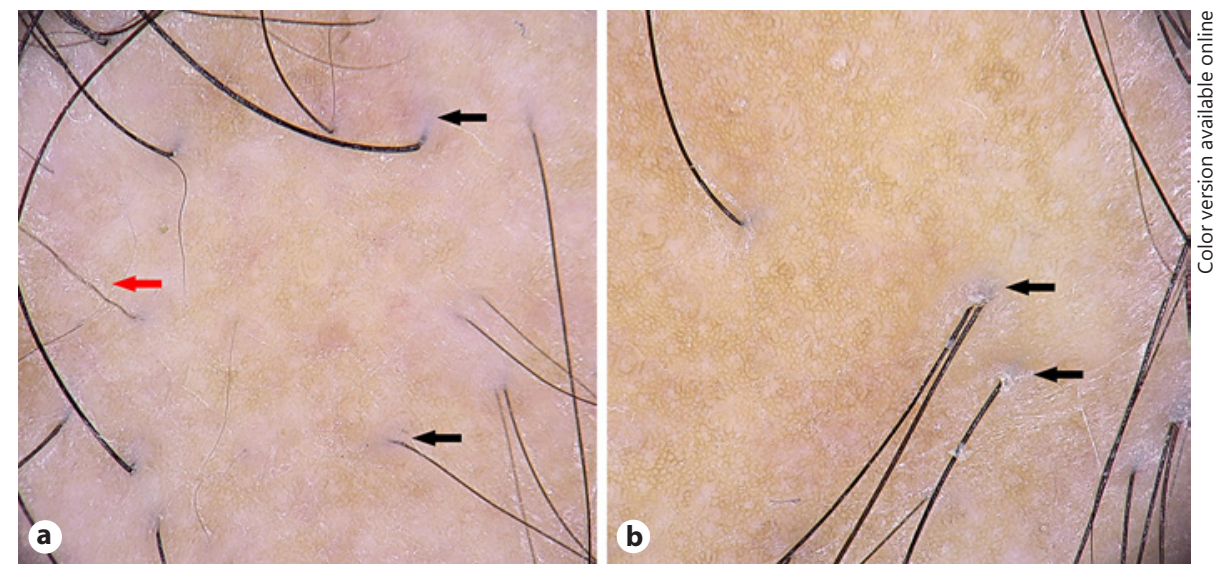

hair line [8]. Three unusual presentations have been identified as well, including (1) androgenetic alopecia-like pattern, (2) cockade-like pattern, and (3) ophiasis-like pattern [9]. FFA usually presents with symmetric bilateral involvement, although an asymmetrical irregular presentation has also been reported [10] and observed by one of us as an asymmetric cockade-like pattern (authors' unpublished data). Herein, we report an unusual presentation of FFA in an "upsilon" pattern not previously described.

\section{Case Report}

A 56-year-old woman presented with hair loss on the front and sides of the scalp that started more than 10 years prior and has progressed without hair regrowth. She denied scalp pain or itch and reported loss of arm, leg, and eyebrow hair but preservation of axillary and pubic hair. She endorsed wearing her hair in a high bun previously for many years but otherwise denied any tight hair styling practices. She does not have a history of taking oral contraceptive pills or receiving radiation therapy. Family history was notable for breast cancer in her mother and ovarian cancer in her sister. 
Fig. 3. Histopathology. Frontal fibrosing alopecia: a terminal anagen follicle with lamellar perifollicular fibrosis and lymphocytic lichenoid infiltrate, horizontal section (a) (hematoxylin and eosin. $\times 10$ ). Traction alopecia: at the level of the isthmus, there are intact sebaceous glands with adjacent areas of follicular dropout at sites of preexisting follicles/follicular units (b) (hematoxylin and eosin. $\times 10$ ).
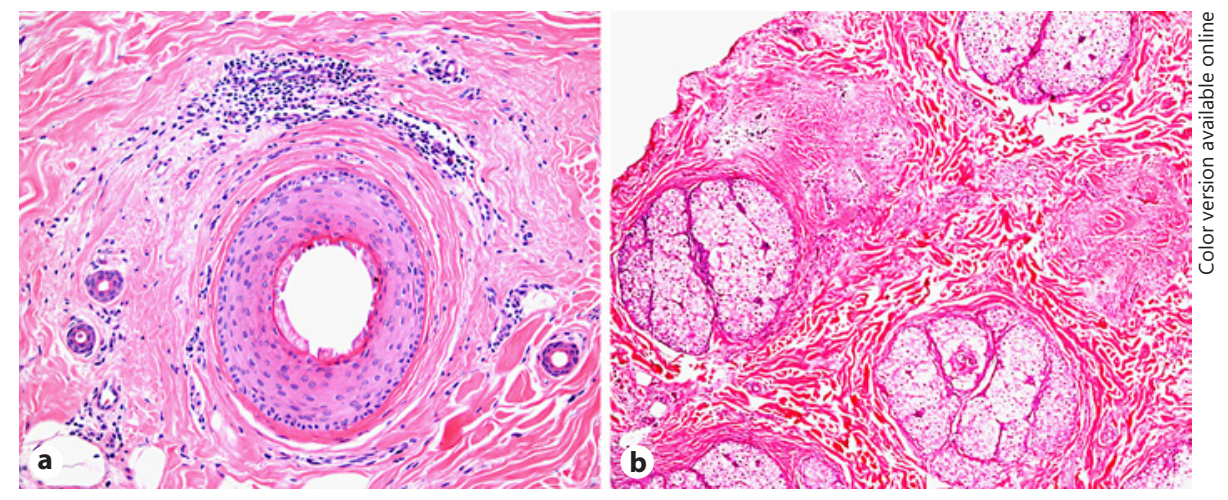

On physical exam, in a middle-aged woman with Fitzpatrick skin type III, there was a smooth band of alopecia along the frontal and temporal hairline extending into 2 symmetrical triangles along the parietal scalp, resembling an upsilon (v)-pattern distribution (Fig. 1). The triangular alopecic patches had their base along the temporal scalp and pointed toward the scalp crown. There was loss of vellus hairs along the frontal hairline and isolated terminal hairs, referred to as lonely hairs, approximately $2 \mathrm{~cm}$ from the actual frontal hairline. She had no hair on all four extremities or eyebrows, and her eyebrows were tattooed. Trichoscopy within the frontal band and the triangular alopecic areas demonstrated loss of follicular openings, terminal hairs with peripilar casts and peripilar hypopigmentation, transparent emergences of the proximal hair shafts, and absence of perifollicular erythema (Fig. 2a, b).

Horizontal sections of a scalp biopsy obtained from the margin of the triangular area with peripilar casts on dermoscopy demonstrated altered follicular architecture with absence of sebaceous glands and decreased follicular density of 20 follicles (13 anagen, 3 telogen, and 4 vellus follicles). One terminal follicle showed perifollicular fibrosis and lichenoid infiltrate (Fig. 3a). A report of a scalp biopsy obtained at an outside facility several years prior noted 3 anagen follicles with infundibular lichenoid lymphoplasmocytic infiltrates, perifollicular fibrosis, and depletion of sebaceous glands. Thus, the histopathology was consistent with a diagnosis of FFA.

The patient was started on topical tacrolimus $0.03 \%$ ointment along with minoxidil 5\% foam to the margins of the hair loss daily. Given her family history of breast cancer, we chose not to prescribe a 5- $\alpha$-reductase inhibitor for safety concerns.

\section{Discussion}

FFA was first described in 1994 by Kossard as a progressive frontotemporal scarring alopecia and thinning of the eyebrows primarily in postmenopausal women [11]. With increased recognition and reporting, the associated features have expanded to include body hair and eyelash loss, facial papules, lonely hairs, glabellar red dots, lichen planus pigmentosus, and depression of the frontal veins [8]. As mentioned, there have been a number of reports of atypical FFA presentations that do not fit the classic description. Atypical presentations of FFA differ from the classic frontotemporal FFA by the distribution of alopecia but maintain the same trichoscopic and histologic findings [8-10]. Regarding prognosis, among the classic types the "pseudo-fringe" pattern has been associated with less progression, whereas the majority of treated patients with the "zig-zag" pattern progressed despite treatment [8]. At this point, it is unclear whether atypical presentations have a different prognosis compared to the classical types due to lack of data.

Our case of FFA with alopecia in a band-like pattern along the frontotemporal scalp extending into 2 symmetrical triangles along the parietal scalp is also unusual/ atypical. We have named this the "upsilon" pattern, mirroring the shape of this letter in the Greek alphabet. To our knowledge, this presentation has not been reported. In addition to scalp hair loss, our patient had lonely hairs and loss of eyebrow and body hair. These clinical, trichoscopic, and histopathologic findings were consistent with a diagnosis of FFA. Our patient did not have perifollicular erythema, which is not always present in FFA, and its absence may indicate disease inactivity [12]. Histology demonstrating perifollicular fibrosis with a lichenoid lymphocytic infiltrate in the absence of sebaceous glands further supports the diagnosis of FFA and excludes traction alopecia or alopecia areata, which may be considered in the differential diagnosis. For comparison, a histologic image of traction alopecia, which was not obtained from our patient, demonstrates intact sebaceous glands at the level of the isthmus with adjacent areas of follicular dropout at sites of preexisting follicles (Fig. 3b). Furthermore, while our patient did endorse a history of wearing tight buns, in traction alopecia due to wearing tight buns (chignon alopecia or traction alopecia in ballerinas) the bulk of trauma is on the entire frontotempo- 
ral scalp or on the occipital scalp (depending on the type of the bun), which leads to hairless patches with a preserved fringe sign $[13,14]$. Our patient does not demonstrate the fringe sign.

Our patient's clinical trajectory is unclear. Further characterization of both typical and atypical FFA presentations and associated treatment responses would allow for better prognostication and management of these challenging patients.

\section{Statement of Ethics}

The authors have no ethical conflicts to disclose. The patient has given her consent to publish photos and details of the case.

\section{Disclosure Statement}

The authors have no conflicts of interest to declare.

\section{Funding Sources}

The authors did not receive any funding.

\section{Author Contributions}

Dr. Goldman and Ms. Diaz wrote the first draft of the manuscript. Dr. Miteva was responsible for acquisition, concept, and design. Dr. Goldman, Ms. Diaz, and Dr. Miteva contributed to analysis, interpretation of data, and critical revision of the article.

\section{References}

1 Iorizzo M, Tosti A. Frontal fibrosing alopecia: an update on pathogenesis, diagnosis, and treatment. Am J Clin Dermatol. 2019 Jun; 20(3):379-90.

2 Lencastre A, Tosti A. Images in clinical medicine. A receding hairline. N Engl J Med. 2013 Jul 11;369(2):e2.

3 Tavakolpour S, Mahmoudi H, Abedini R, Kamyab Hesari K, Kiani A, Daneshpazhooh M. Frontal fibrosing alopecia: an update on the hypothesis of pathogenesis and treatment. Int J Womens Dermatol. 2019 Jun;5(2):116-23.

4 Tziotzios C, Stefanato CM, Fenton DA, Simpson MA, McGrath JA. Frontal fibrosing alopecia: reflections and hypotheses on aetiology and pathogenesis. Exp Dermatol. 2016 Nov; 25(11):847-52.

5 Tziotzios C, Petridis C, Dand N, Ainali C, Saklatvala JR, Pullabhatla V, et al. Genomewide association study in frontal fibrosing alopecia identifies four susceptibility loci including HLA-B*07:02. Nat Commun. 2019 Mar 8;10(1):1150.
6 Starace M, Brandi N, Alessandrini A, Bruni F, Piraccini BM. Frontal fibrosing alopecia: a case series of 65 patients seen in a single Italian centre. J Eur Acad Dermatol Venereol. 2019 Feb;33(2):433-8.

7 Tosti A, Miteva M, Torres F. Lonely hair: a clue to the diagnosis of frontal fibrosing alopecia. Arch Dermatol. 2011 Oct; 147(10):1240.

8 Moreno-Arrones OM, Saceda-Corralo D, Fonda-Pascual P, Rodrigues-Barata AR, Buendía-Castaño D, Alegre-Sánchez A, et al. Frontal fibrosing alopecia: clinical and prognostic classification. J Eur Acad Dermatol Venereol. 2017 Oct;31(10):1739-45.

9 Rossi A, Grassi S, Fortuna MC, Garelli V, Pranteda G, Caro G, et al. Unusual patterns of presentation of frontal fibrosing alopecia: a clinical and trichoscopic analysis of $98 \mathrm{pa}-$ tients. J Am Acad Dermatol. 2017 Jul;77(1): 172-4.
10 Contin LA, de Almeida Ledá YL, Caldeira Nassif K, Suárez Restrepo MV. Patchy frontal fibrosing alopecia: description of an incomplete clinical presentation. Skin Appendage Disord. 2017 Oct;3(4):190-2.

11 Kossard S. Postmenopausal frontal fibrosing alopecia. Scarring alopecia in a pattern distribution. Arch Dermatol. 1994 Jun;130(6):7704.

12 Toledo-Pastrana T, Hernández MJ, Camacho Martínez FM. Perifollicular erythema as a trichoscopy sign of progression in frontal fibrosing alopecia. Int J Trichology. $2013 \mathrm{Jul}$ 5(3):151-3.

13 Trueb RM. "Chignon alopecia": a distinctive type of nonmarginal traction alopecia. Cutis. 1995 Mar;55(3):178-9.

14 Samrao A, Chen C, Zedek D, Price VH. Traction alopecia in a ballerina: clinicopathologic features. Arch Dermatol. 2010 Aug;146(8): 930-1. 